\title{
Deeply Subwavelength Localization with Reverberation-Coded Aperture
}

\author{
Michael del Hougne $\odot,{ }^{1}$ Sylvain Gigan $\odot,{ }^{2}$ and Philipp del Hougne $\odot^{3, *}$ \\ ${ }^{1}$ Julius-Maximilians-Universität Würzburg, D-97070 Würzburg, Germany \\ ${ }^{2}$ Laboratoire Kastler Brossel, Université Pierre et Marie Curie, Ecole Normale Supérieure, \\ CNRS, Collège de France, F-75005 Paris, France \\ ${ }^{3}$ Univ Rennes, CNRS, IETR - UMR 6164, F-35000 Rennes, France
}

(Received 10 February 2021; revised 10 June 2021; accepted 14 June 2021; published 23 July 2021)

\begin{abstract}
Accessing subwavelength information about a scene from the far-field without invasive near-field manipulations is a fundamental challenge in wave engineering. Yet it is well understood that the dwell time of waves in complex media sets the scale for the waves' sensitivity to perturbations. Modern coded-aperture imagers leverage the degrees of freedom (d.o.f.) offered by complex media as natural multiplexor but do not recognize and reap the fundamental difference between placing the object of interest outside or within the complex medium. Here, we show that the precision of localizing a subwavelength object can be improved by several orders of magnitude simply by enclosing it in its far field with a reverberant passive chaotic cavity. We identify deep learning as a suitable noise-robust tool to extract subwavelength localization information encoded in multiplexed measurements, achieving resolutions well beyond those available in the training data. We demonstrate our finding in the microwave domain: harnessing the configurational d.o.f. of a simple programmable metasurface, we localize a subwavelength object along a curved trajectory inside a chaotic cavity with a resolution of $\lambda / 76$ using intensity-only single-frequency single-pixel measurements. Our results may have important applications in photoacoustic imaging as well as humanmachine interaction based on reverberating elastic waves, sound, or microwaves.
\end{abstract}

DOI: 10.1103/PhysRevLett.127.043903

Retrieving a representation of an object based on how it scatters waves is a central goal across all areas of wave engineering (light, microwaves, sound,...) with applications ranging from biomedicine via microelectronics to astrophysics. Since wave energy cannot, in general, be focused beyond the diffraction limit in free space, a widespread misconception is that subwavelength information can only be accessed via evanescent waves. This argument ignores the crucial roles of a priori knowledge and signal-to-noise ratio (SNR); moreover, many imaging schemes do not even rely on focusing. Indeed, given extensive a priori knowledge, an imaging task can collapse to a curve fitting exercise without any fundamental bound on the achievable precision (e.g., deconvolution microscopy [1,2]). The advent of deep learning has enabled elaborate demonstrations of such nonlinear function approximations, facilitating deeply subwavelength imaging even with a simple plane wave from the far field [3]. Despite the resulting frequent absence of any wavelength-induced fundamental resolution bounds, specific physical mechanisms can be

Published by the American Physical Society under the terms of the Creative Commons Attribution 4.0 International license. Further distribution of this work must maintain attribution to the author(s) and the published article's title, journal citation, and DOI. useful to boost the practically achievable resolution. A common example is the above-mentioned access to evanescent waves either via near-field measurements $[4,5]$ or by coupling them to the far field with near-field scatterers [615]. Similarly to the use of fluorescent markers [16], these approaches are inherently invasive since they rely on manipulations of the object's near field. A further notable idea relies on tailored coherent far-field illumination to create superoscillatory hotspots $[17,18]$ but suffers from inherently low SNRs.

In the wave chaos community [19], it is well known that a wave's sensitivity to geometrical perturbations [20-23] is directly related to its dwell time in the interaction domain [24]. This effect can be thought of as a generalization of the subwavelength interferometric sensitivity in phase microscopy [25-27] or high-finesse Fabry-Perot cavities [28]. If the scene to be imaged is enclosed in its far field by a reverberant chaotic cavity, the dwell time is drastically enhanced. Different scenes can then be interpreted as different perturbations of an otherwise static complex scattering geometry. The extreme sensitivity of linear passive wave chaos has been leveraged to detect the presence of minute changes and to characterize their global features (e.g., scattering cross section), notably in diffusing wave spectroscopy [23,29-31]. However, its potential to significantly improve the resolution with which a subwavelength perturber can be localized without any near-field 
manipulation has remained unexplored, presumably because of the postprocessing challenge to untangle the complete scrambling of waves (and the information that they carry) inside the complex medium. But it is also widely assumed that the localization resolution in wavechaotic systems is inherently diffraction limited [32,33], unless a nonlinear feedback loop involving gain and giving rise to self-oscillations is used [34].

At the same time, rich literature exists on imaging and sensing with a complex medium as coded aperture (CA). This research track is driven by the desire to achieve imaging with as few detectors and measurements as possible. Rather than directly mapping the object to its image, the spatial object information can be multiplexed across random configurations of a CA onto a single detector [35] - see Fig. 1(a). Practical implementations of CAs often leverage the fact that wave transmission through a complex medium (multiply scattering medium, chaotic cavity, disordered metamaterial) constitutes random multiplexing thanks to the mediums spectral, spatial or configurational degrees of freedom (d.o.f.) [36-40]Fig. 1(b) illustrates the former. In other words, the transmission matrix of a complex medium naturally offers the desired properties of a random multiplexing matrix [36,38].

Within this realm, scenarios in which object and wave source are embedded within the complex medium, as in Fig. 1(c), have been treated as a simple alternative way of

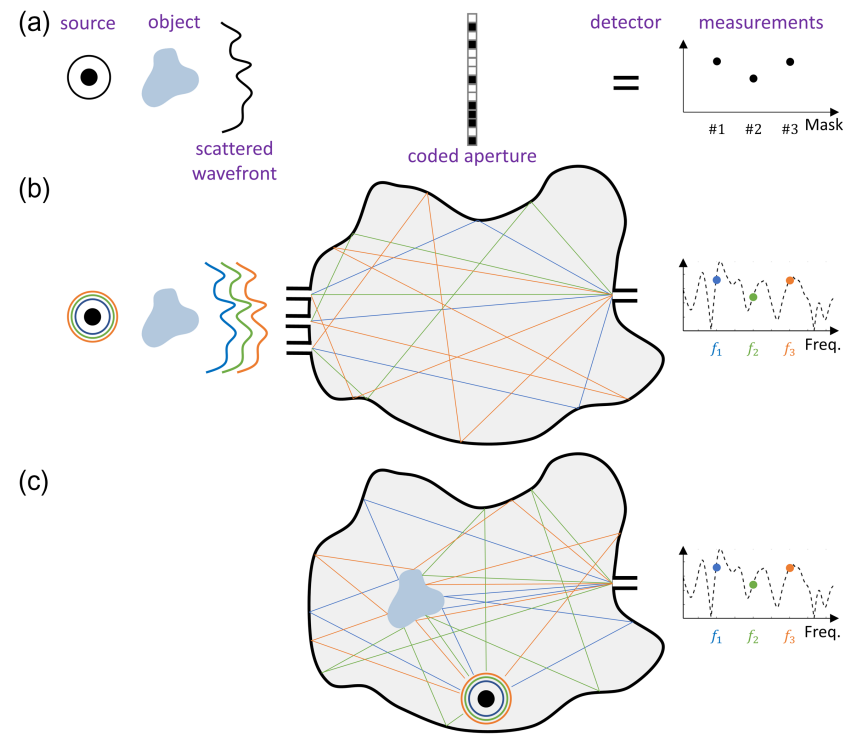

FIG. 1. (a) Conventional CA. A wavefront is scattered by an object and then multiplexed across different masks onto a singlepixel detector. (b) Use of a complex medium's spectral d.o.f. (color coded) as CA. Wavefronts are scattered by an object and then propagate through a chaotic cavity such that spatial information is multiplexed across different frequencies captured by a single-pixel detector. (c) Reverberation-coded aperture: same as (b) except that object and wave source are inside the chaotic cavity. natural random multiplexing. In photoacoustics, it was recently suggested to enclose the imaging target in an acoustically reverberant cavity [41]. Moreover, several schemes for human-machine interaction are inevitably confronted with waves reverberating around an object, for instance, object localization with microwaves in indoor environments or with elastic waves in solid plates [32,33,42-44]. However, the benefits of such "reverberation-coded apertures" (RCAs) go far beyond randomized multiplexing. If the object is inside (rather than outside) the complex medium, the wave interacts with the object not once but countless times, thereby developing a much higher sensitivity to subwavelength object details. Recent efforts to construct optimal coherent states for sensing in complex media $[45,46]$ differ from our problem, besides their requirement for multichannel excitation, in that they rely on (and are limited to) small perturbations of the soughtafter variable.

In this Letter, we introduce RCAs, combined with deep learning to extract encoded subwavelength localization information, as a truly noninvasive route to deeply subwavelength localization resolution. We first establish the fundamental link between dwell time, wave sensitivity, and localization resolution in a semi-analytical study of the prototypical example of object localization along a curved trajectory (i.e., one coordinate is unknown) inside a passive chaotic cavity using spectral d.o.f. Then, we demonstrate our finding experimentally in the microwave domain, using configurational d.o.f. provided by a simple programmable metasurface.

To start, we consider for concreteness a 2D model problem in semianalytical simulations based on a coupled-dipole formalism (see Refs. [15,47] and the Supplemental Material [48]). As depicted in Figs. 2(a)2(c), we consider three scenarios expected to correspond to different durations of the scattering process: free space, a cavity with quality factor $Q=263$ and a cavity with $Q=556$. In each case, the transmission $S_{12}$ between a transmit and a receive port is measured for various frequencies in order to localize [using artificial neural network (ANN)-based data analysis] a nonresonant dipole that could be located anywhere along a circular perimeter. Given the single-channel nature of our single-detector scheme, we estimate the duration of the scattering process via the "phase delay time" $\tau=\partial \arg \left(S_{12}\right) / \partial \omega$ [53]. The irregularly shaped cavity constitutes a complex medium for wave propagation in which $\tau$ is hence a statistically distributed quantity; in Fig. 2(d), we plot the cumulative distribution function (CDF) of its magnitude for the three considered cases, confirming that they correspond to increasing dwell times. Compared to a regular cavity, such a chaotic cavity has not only the practical advantage of being easy to implement but also that ergodicity ensures statistically similar properties $[54,55]$ irrespective of the object position. 

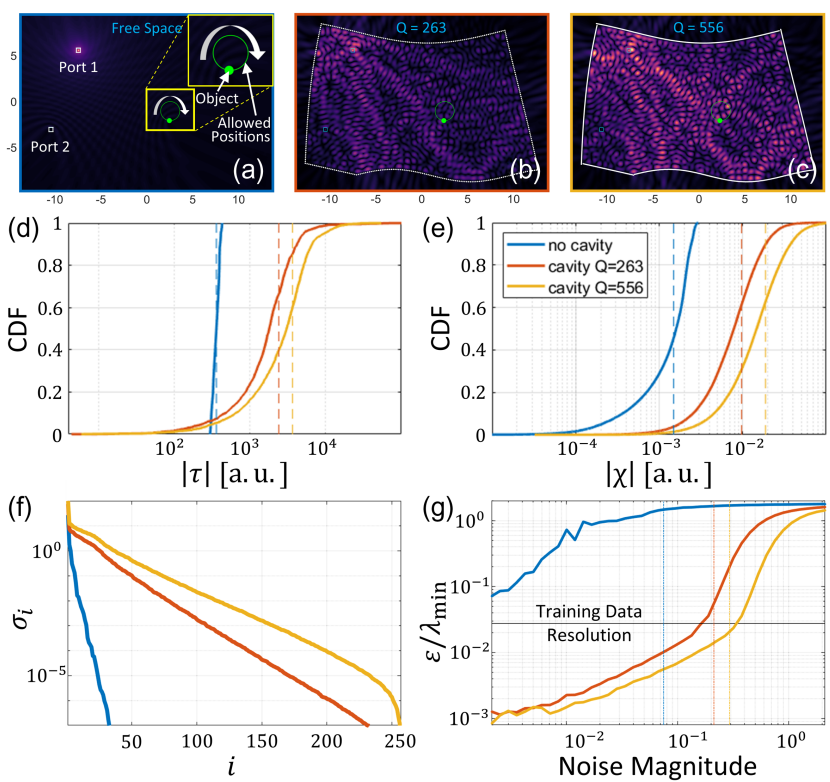

$|\chi|[$ a.u. $]$

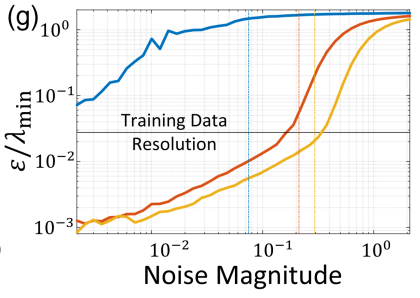

FIG. 2. (a)-(c) Electric field magnitude in 2D semianalytical simulations for (a) free space, a chaotic cavity with (b) $Q=263$, and (c) $Q=556$. All maps use the same color scale. (d) CDF of the dwell time magnitude $|\tau|$ distribution in the three cases. Vertical dashed lines indicate the corresponding mean values. (e) $\mathrm{CDF}$ of the parametric derivative magnitude $|\chi|$ (with respect to the object position) in the three cases. Vertical dashed lines indicate the corresponding mean values. (f) SVs $\sigma_{i}$ of $\mathbf{T}(X, f)$ (without any noise) for the three considered cases. (g) Average localization error $\epsilon$ in terms of the smallest utilized wavelength $\lambda_{\min }$ as a function of the absolute magnitude of the measurement noise for the three cases. Vertical dashed lines indicate the corresponding signal magnitudes. The horizontal black line indicates the training data resolution.

The dwell time plays a crucial role in mesoscopic physics because it is related to several other relevant quantities [53,56-58], some of which happen to also be critical metrics for RCA-based imaging and sensing. The most obvious quantity is the energy stored in the complex medium $[53,59,60]$ which is directly related to the received signal strength and thereby the measurement's SNR (assuming detector-induced noise). Of course, measurements with higher SNR contain more information. The direct link between $|\tau|$ and the stored energy is apparent upon visual inspection of Figs. 2(a)-2(c), and indeed the received signal strength is on average 2.9 [3.9] times higher in (b) [(c)] than in (a).

The sensitivity to parametric perturbations of the scattering system is another important quantity that can be related to the wave's dwell time in the interaction domain [24,61]. Intuitively, this can be understood as follows: the probability that a tiny perturbation impacts the evolution of a wave increases with the wave's lifetime in the interaction domain. Analogous to $\tau$, we define $\chi=\partial S_{12} / \partial X$, where $X$ denotes the considered parameter (the object position along the allowed trajectory in our case). Indeed, in Figs. 2(d), 2 (e) we observe a clear correspondence between the distributions of $|\tau|$ and $|\chi|$, for instance, in terms of the mean value (dashed vertical lines). The difference between two nearby object positions in terms of the corresponding scattering matrices (specifically, $S_{12}$ ) is thus on average larger if the dwell time in the interaction domain is larger. This effect is induced by using a RCA instead of a conventional $\mathrm{CA}$ for which the object scatters the wave before the wave is multiplexed across the CA.

In the case of a CA (RCA or conventional) leveraging spectral d.o.f., the amount of information that can be extracted from measurements within a given bandwidth is also tightly linked to the dwell time. Indeed, the spectral decorrelation is related to the rate at which $S_{12}$ fluctuates with respect to the frequency. The lower the correlation between different measurement modes is, the less redundant information is acquired. To illustrate this effect, we consider the singular value (SV) decomposition of a 2D matrix $\mathbf{T}(X, f)$ containing the measured transmission for different frequencies and positions along the allowed trajectory; $\mathbf{T}(X, f)$ later serves as training data for the ANN. In Fig. 2(f), we plot the SV spectra of this matrix for all three considered cases. Indeed, higher dwell times correlate with a flatter SV spectrum, implying that different frequencies are less correlated.

Having established three distinct RCA mechanisms expected to enhance the possibility of physically encoding deeply subwavelength information about the object via wave propagation inside the RCA in multiplexed measurements, we now tackle the problem of digitally decoding this information. In order to approximate an inverse function of the physical wave scattering process, mapping the measured data to the object position, we use deep learning. We deliberately use an ANN consisting of several fully connected layers (see Supplemental Material [48]) as opposed to more popular convolutional architectures because the latter excel at identifying relevant local correlations in the data whereas we hypothesize that the complete scrambling caused by wave scattering encodes the relevant features in long-range correlations within the data $[47,62,63]$. Moreover, our ANN does not solve a classification problem but predicts a continuous variable: the object's position.

We report in Fig. 2(g) the average localization error in terms of the smallest utilized wavelength, $\epsilon / \lambda_{\min }$, as a function of the measurement noise magnitude. First, we observe that even the free space scenario can achieve deeply subwavelength resolution beyond $\lambda_{\min } / 10$ at low noise levels, stressing the absence of any fundamental wavelength-induced resolution bounds, similar to Refs. [13]. Second, as hypothesized, the longer the dwell time in the RCA, the higher the achievable resolution at a given noise level. In our case, we observe resolutions beyond $\lambda_{\min } / 10^{3}$ but, as justified above, we refrain from comparing these absolute resolution values to other works with different a priori knowledge and SNR. Third, remarkably, 
the achievable resolution can be more than an order of magnitude better than the resolution of the training data, suggesting that beyond being an efficient approximator to "seen" data, our ANN also very faithfully interpolates between seen data points. Deep learning also offers a remarkable noise robustness which significantly outperforms a simple multivariate linear regression (see Supplemental Material [48] Sec. D and Fig. S3).

One question naturally arises: can we isolate the contribution of the three identified RCA mechanisms? Specifically, we now evidence the major role of the dwell-time-enhanced sensitivity to tiny perturbations. To that end, we consider a setting in which the other two factors do not impact the localization accuracy: we compare the three considered scenarios in terms of their SNR (removing benefits due to enhanced signal strength), and operate with a single d.o.f. (removing benefits due to faster spectral decorrelation). Table I summarizes the achievable localization accuracies for two rather high values of SNR; the focus here is not on the absolute localization precision but on how it compares between the three considered scenarios. The dependence of the achieved localization accuracy on the dwell time emerges very clearly, confirming our argument that placing the scene inside a RCA rather than outside a CA boosts the sensitivity to subwavelength scene details. Incidentally, even in Table I we observe a resolution of $\sim \lambda / 3.8$ for the RCA with $Q=556$ at an SNR of $30 \mathrm{~dB}$.

Having investigated fundamental RCA mechanisms, we now report an experimental demonstration in the microwave domain inside a 3D irregularly shaped metallic enclosure-see Fig. 3(a). We use configurational instead of spectral d.o.f.: we measure the transmission between two antennas ("single-pixel detector") at a single frequency $\left(f_{0}=2.463 \mathrm{GHz}\right)$ but for a fixed series of random configurations (parameter $c$ ) of the cavity's scattering properties. The latter is conveniently implemented with a simple programmable metasurface $[64,65]$ consisting of an array of individually tunable meta-atoms with two digitalized states mimicking Dirichlet or Neumann boundary conditions [66]. Moreover, we now only use the intensity information of the measurements, to illustrate that RCAs enable deeply subwavelength resolution even without access to phase information, which relaxes hardware requirements considerably. We access different dwell time regimes by tuning the opening of the cavity's ceiling.

TABLE I. Average localization error $\epsilon / \lambda_{\min }$ using a single d.o.f. for two magnitudes of the measurement noise relative to the measured signal strength.

\begin{tabular}{lccc}
\hline \hline SNR $[\mathrm{dB}]$ & No cavity & Cavity $Q=263$ & Cavity $Q=556$ \\
\hline 30 & 1.12 & 0.53 & 0.26 \\
60 & 0.59 & 0.31 & 0.24 \\
\hline \hline
\end{tabular}

Of the above identified three distinct RCA mechanisms, two (signal strength and sensitivity) are independent of the utilized type of d.o.f.; the third (measurement diversity) turns out to be favorably linked to enhanced dwell times if configurational d.o.f. are used, too, albeit for a different reason. The amount of information that can be extracted from a series of measurements at $f_{0}$ with random metasurface configurations is larger if the latter induce stronger fluctuations of $S_{12}\left(f_{0}\right)$. A longer dwell time correlates with a larger standard deviation of $\left|S_{12}\left(f_{0}\right)\right|$, as evidenced in Fig. 3(b). This can be understood by decomposing the transmission between the two ports into all contributing ray paths. If the metasurface is small compared to the cavity surface and the dwell time is relatively low, only a few rays are affected by the metasurface configuration. We sketch for such a scenario the cloud of accessible $S_{12}\left(f_{0}\right)$ values in
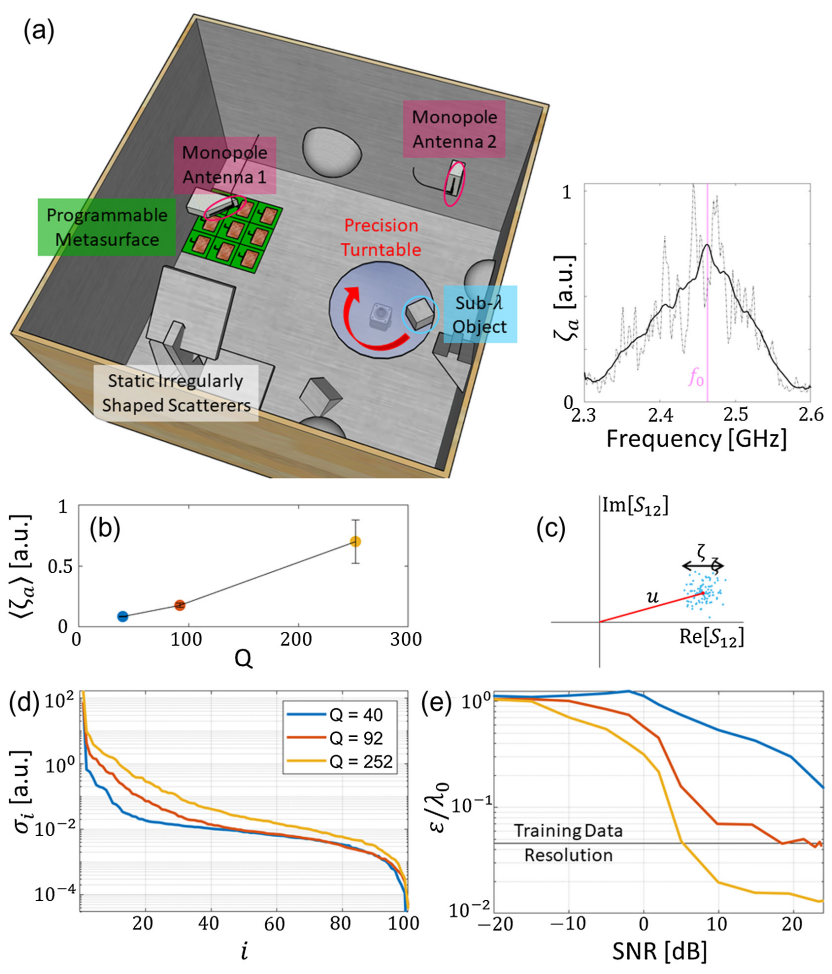

FIG. 3. (a) Experimental setup: a 3D complex scattering enclosure contains a subwavelength metallic object on a precision turntable, two monopole antennas, and a programmable metasurface in the vicinity of one antenna. The enclosure's ceiling can be open $(Q=40)$, partially covered $(Q=92)$, or fully covered $(Q=252)$ with metal. See Supplemental Material [48] for technical details. The inset shows the standard deviation $\zeta_{a}$ of $\left|S_{12}\right|$ over 100 random metasurface configurations and identifies the chosen operating frequency $f_{0}$. (b) Dependence of mean of $\zeta_{a}$ over object positions on $Q$. (c) Sketch of $S_{12}\left(f_{0}\right)$ distribution for 100 random metasurface configurations. (d) SV spectra of $\mathbf{T}(X, c)$ for the three considered cases. (e) Average localization error $\epsilon$ in terms of the utilized wavelength $\lambda_{0}$ as a function of SNR. The horizontal black line indicates the training data resolution. 
the Argand diagram in Fig. 3(c); the cloud is not centered on the origin because many rays are not controlled by the metasurface. The longer the dwell time, the larger the percentage of rays that encounter some of the metasurface elements such that the radius of the cloud increases, as witnessed in Fig. 3(b). To illustrate that $\mathbf{T}(X, c)$ contains more information if the dwell time is longer, we plot the corresponding SV spectra in Fig. 3(d). A qualitatively similar trend as in Fig. 2(f) is seen, despite the use of a different type of d.o.f.

The average experimental localization error plotted in Fig. 3(e) is consequently significantly lower if the dwell time is longer. The dependence on the SNR is evaluated by adding white noise to the experimentally measured values. Unlike in Fig. 2(g), we plot $\epsilon / \lambda_{0}$ as a function of the relative rather than absolute noise magnitude due to experimental constraints, such that the curve does not reflect the first RCA mechanism's benefits (signal strength). Despite the use of low-cost measurement equipment (see Supplemental Material [48]) and intensity-only data, we achieve resolutions up to $\lambda / 76$ in our experiment. For $Q=252$, we observe once again that our ANN decoder achieves a resolution clearly exceeding that of the training data.

To conclude, in this Letter, we proved that reverberation in a passive complex medium efficiently encodes deeply subwavelength details in multiplexed measurements without any manipulation of the object's near field. We evidenced that the wave's dwell time is directly linked to the achievable localization resolution via three mechanisms, irrespective of the utilized type of d.o.f.: (i) enhanced signal strength, (ii) enhanced sensitivity, and (iii) enhanced measurement diversity. We further showed that ANNs are capable of decoding such measurements with unexpectedly high fidelity. In microwave experiments in a chaotic cavity leveraging the configurational d.o.f. offered by a programmable metasurface, we successfully localized subwavelength objects on curved trajectories with a resolution of $\lambda / 76$.

Looking forward, evaluating potential benefits of using a self-oscillating source $[34,67]$ that operates at a real-valued pole associated with a diverging delay time is a first avenue for future exploration. We also envision a "learned RCA" that jointly optimizes physical encoding and digital decoding in an end-to-end fashion [68], and may rely on learned models of the physical layer [69,70]. Moreover, our method can be straightforwardly applied to multidimensional localization and conceptual extensions to more complex imaging tasks are conceivable, e.g., to recognize subwavelength object shapes or materials inside a RCA without any near-field manipulation. In a more applied context, various single-element detection methods, including RCAs, are currently being explored in photoacoustic imaging [41,71,72]; our work encourages investigating whether photoacoustic imaging can capitalize on potential resolution enhancements offered by the RCA but not by other "ergodic relays." Further practical applications lie in emerging techniques for human-machine interactions which naturally deal with reverberating waves of different types [42-44]. In the optical domain, our scheme may be applied to the deeply subwavelength localization of microscopic particles, by sandwiching them between two multiply-scattering slabs such as white paint or biological tissue [73].

*philipp.delhougne@gmail.com

[1] J.-B. Sibarita, Deconvolution Microscopy (Springer, New York, 2005).

[2] P. Sarder and A. Nehorai, Deconvolution methods for 3-d fluorescence microscopy images, IEEE Signal Process. Mag. 23, 32 (2006).

[3] T. Pu, J.-Y. Ou, N. Papasimakis, and N. I. Zheludev, Labelfree deeply subwavelength optical microscopy, Appl. Phys. Lett. 116, 131105 (2020).

[4] E. Betzig and R. J. Chichester, Single molecules observed by near-field scanning optical microscopy, Science 262, 1422 (1993).

[5] E. Seo, Y.-H. Jin, W. Choi, Y. Jo, S. Lee, K.-D. Song, J. Ahn, Q.-H. Park, M.-K. Kim, and W. Choi, Near-field transmission matrix microscopy for mapping high-order eigenmodes of subwavelength nanostructures, Nat. Commun. 11, 2575 (2020).

[6] J. B. Pendry, Negative Refraction makes a Perfect Lens, Phys. Rev. Lett. 85, 3966 (2000).

[7] N. Fang, H. Lee, C. Sun, and X. Zhang, Sub-diffractionlimited optical imaging with a silver superlens, Science 308, 534 (2005).

[8] S. Guenneau, A. Movchan, G. Pétursson, and S. A. Ramakrishna, Acoustic metamaterials for sound focusing and confinement, New J. Phys. 9, 399 (2007).

[9] A. Sukhovich, B. Merheb, K. Muralidharan, J. O. Vasseur, Y. Pennec, P. A. Deymier, and J. H. Page, Experimental and Theoretical Evidence for Subwavelength Imaging in Phononic Crystals, Phys. Rev. Lett. 102, 154301 (2009).

[10] F. Lemoult, G. Lerosey, J. de Rosny, and M. Fink, Resonant Metalenses for Breaking the Diffraction Barrier, Phys. Rev. Lett. 104, 203901 (2010).

[11] E. G. van Putten, D. Akbulut, J. Bertolotti, W. L. Vos, A. Lagendijk, and A. P. Mosk, Scattering Lens Resolves Sub$100 \mathrm{~nm}$ Structures with Visible Light, Phys. Rev. Lett. 106, 193905 (2011).

[12] J. Zhu, J. Christensen, J. Jung, L. Martin-Moreno, X. Yin, L. Fok, X. Zhang, and F. Garcia-Vidal, A holey-structured metamaterial for acoustic deep-subwavelength imaging, Nat. Phys. 7, 52 (2011).

[13] J.-H. Park, C. Park, H. Yu, J. Park, S. Han, J. Shin, S. H. Ko, K. T. Nam, Y.-H. Cho, and Y. Park, Subwavelength light focusing using random nanoparticles, Nat. Photonics 7, 454 (2013).

[14] C. Park, J.-H. Park, C. Rodriguez, H. S. Yu, M. Kim, K. Jin, S. Han, J. Shin, S. H. Ko, K. T. Nam et al., Full-Field Subwavelength Imaging Using a Scattering Superlens, Phys. Rev. Lett. 113, 113901 (2014). 
[15] B. Orazbayev and R. Fleury, Far-Field Subwavelength Acoustic Imaging by Deep Learning, Phys. Rev. X 10, 031029 (2020).

[16] S. W. Hell and J. Wichmann, Breaking the diffraction resolution limit by stimulated emission: Stimulated-emissiondepletion fluorescence microscopy, Opt. Lett. 19, 780 (1994).

[17] E. T. Rogers, J. Lindberg, T. Roy, S. Savo, J. E. Chad, M. R. Dennis, and N. I. Zheludev, A super-oscillatory lens optical microscope for subwavelength imaging, Nat. Mater. 11, 432 (2012).

[18] M. Dubois, E. Bossy, S. Enoch, S. Guenneau, G. Lerosey, and P. Sebbah, Time-Driven Superoscillations with Negative Refraction, Phys. Rev. Lett. 114, 013902 (2015).

[19] H.-J. Stöckmann, Quantum Chaos: An Introduction (Cambridge University Press, Cambridge, England, 1999).

[20] T. Gorin, T. Prosen, T. H. Seligman, and M. Žnidarič, Dynamics of Loschmidt echoes and fidelity decay, Phys. Rep. 435, 33 (2006).

[21] U. Kuhl, Microwave experiments in the realm of fidelity, Phil. Trans. R. Soc. A 374, 20150158 (2016).

[22] B. T. Taddese, T. M. Antonsen, E. Ott, and S. M. Anlage, Sensing small changes in a wave chaotic scattering system, J. Appl. Phys. 108, 114911 (2010).

[23] B. T. Taddese, G. Gradoni, F. Moglie, T. M. Antonsen, E. Ott, and S.M. Anlage, Quantifying volume changing perturbations in a wave chaotic system, New J. Phys. 15, 023025 (2013).

[24] P. W. Brouwer, K. M. Frahm, and C. W. J. Beenakker, Quantum Mechanical Time-Delay Matrix in Chaotic Scattering, Phys. Rev. Lett. 78, 4737 (1997).

[25] Y. Park, C. Depeursinge, and G. Popescu, Quantitative phase imaging in biomedicine, Nat. Photonics 12, 578 (2018).

[26] T. Juffmann, A. de los Ríos Sommer, and S. Gigan, Local optimization of wave-fronts for optimal sensitivity phase imaging (lowphi), Opt. Commun. 454, 124484 (2020).

[27] D. Bouchet, J. Dong, D. Maestre, and T. Juffmann, Fundamental Bounds on the Precision of Classical Phase Microscopes, Phys. Rev. Applied 15, 024047 (2021).

[28] O. Arcizet, P.-F. Cohadon, T. Briant, M. Pinard, A. Heidmann, J.-M. Mackowski, C. Michel, L. Pinard, O. Français, and L. Rousseau, High-Sensitivity Optical Monitoring of a Micromechanical Resonator with a QuantumLimited Optomechanical Sensor, Phys. Rev. Lett. 97, 133601 (2006).

[29] D. J. Pine, D. A. Weitz, P. M. Chaikin, and E. Herbolzheimer, Diffusing Wave Spectroscopy, Phys. Rev. Lett. 60, 1134 (1988).

[30] G. Maret, Diffusing-wave spectroscopy, Curr. Opin. Colloid Interface Sci. 2, 251 (1997).

[31] J. de Rosny, P. Roux, M. Fink, and J. H. Page, Field Fluctuation Spectroscopy in a Reverberant Cavity with Moving Scatterers, Phys. Rev. Lett. 90, 094302 (2003).

[32] P. del Hougne, M. F. Imani, M. Fink, D. R. Smith, and G. Lerosey, Precise Localization of Multiple Noncooperative Objects in a Disordered Cavity by Wave Front Shaping, Phys. Rev. Lett. 121, 063901 (2018).

[33] R. K. Ing, N. Quieffin, S. Catheline, and M. Fink, In solid localization of finger impacts using acoustic time-reversal process, Appl. Phys. Lett. 87, 204104 (2005).
[34] S. D. Cohen, H. L. D. de S. Cavalcante, and D. J. Gauthier, Subwavelength Position Sensing Using Nonlinear Feedback and Wave Chaos, Phys. Rev. Lett. 107, 254103 (2011).

[35] W. L. Chan, K. Charan, D. Takhar, K. F. Kelly, R. G. Baraniuk, and D. M. Mittleman, A single-pixel terahertz imaging system based on compressed sensing, Appl. Phys. Lett. 93, 121105 (2008).

[36] A. Liutkus, D. Martina, S. Popoff, G. Chardon, O. Katz, G. Lerosey, S. Gigan, L. Daudet, and I. Carron, Imaging with nature: Compressive imaging using a multiply scattering medium, Sci. Rep. 4, 5552 (2014).

[37] J. Hunt, T. Driscoll, A. Mrozack, G. Lipworth, M. Reynolds, D. Brady, and D. R. Smith, Metamaterial apertures for computational imaging, Science 339, 310 (2013).

[38] T. Fromenteze, O. Yurduseven, M. F. Imani, J. Gollub, C. Decroze, D. Carsenat, and D. R. Smith, Computational imaging using a mode-mixing cavity at microwave frequencies, Appl. Phys. Lett. 106, 194104 (2015).

[39] Y. Xie, T.-H. Tsai, A. Konneker, B.-I. Popa, D. J. Brady, and S. A. Cummer, Single-sensor multispeaker listening with acoustic metamaterials, Proc. Natl. Acad. Sci. U.S.A. 112, 10595 (2015).

[40] T. Sleasman, M. F. Imani, J. N. Gollub, and D. R. Smith, Dynamic metamaterial aperture for microwave imaging, Appl. Phys. Lett. 107, 204104 (2015).

[41] M. Brown, E. Zhang, B. Treeby, P. Beard, and B. Cox, Reverberant cavity photoacoustic imaging, Optica 6, 821 (2019).

[42] A. Bourdoux, A. N. Barreto, B. van Liempd, C. de Lima, D. Dardari, D. Belot, E.-S. Lohan, G. Seco-Granados, H. Sarieddeen, H. Wymeersch et al., 6G white paper on localization and sensing, arXiv:2006.01779.

[43] G. C. Alexandropoulos, N. Shlezinger, and P. del Hougne, Reconfigurable intelligent surfaces for rich scattering wireless communications: Recent experiments, challenges, and opportunities, IEEE Commun. Mag. 59, 28 (2021).

[44] Y. Liu, J.-P. Nikolovski, N. Mechbal, M. Hafez, and M. Vergé, Tactile objects based on an amplitude disturbed diffraction pattern method, Appl. Phys. Lett. 95, 251904 (2009).

[45] D. Bouchet, R. Carminati, and A. P. Mosk, Influence of the Local Scattering Environment on the Localization Precision of Single Particles, Phys. Rev. Lett. 124, 133903 (2020).

[46] D. Bouchet, S. Rotter, and A. P. Mosk, Maximum information states for coherent scattering measurements, Nat. Phys. 17, 564 (2021).

[47] P. del Hougne, Robust position sensing with wave fingerprints in dynamic complex propagation environments, Phys. Rev. Research 2, 043224 (2020).

[48] See Supplemental Material at http://link.aps.org/ supplemental/10.1103/PhysRevLett.127.043903 for details on the semianalytical simulations, the experimental setup, the ANN architecture, a comparison between linear multivariable regression and deep learning, the normalized parametric derivative distributions, and further parameters that impact the localization precision, which includes Refs. [49-52].

[49] Myriad-RF, LMS7002M Python package (2019). 
[50] D. P. Kingma and J. Ba, Adam: A method for stochastic optimization, arXiv:1412.6980.

[51] P. del Hougne, M. Fink, and G. Lerosey, Optimally diverse communication channels in disordered environments with tuned randomness, Nat. Electron. 2, 36 (2019).

[52] P. del Hougne, M. Davy, and U. Kuhl, Optimal Multiplexing of Spatially Encoded Information across Custom-Tailored Configurations of a Metasurface-Tunable Chaotic Cavity, Phys. Rev. Applied 13, 041004(R) (2020).

[53] S. Rotter and S. Gigan, Light fields in complex media: Mesoscopic scattering meets wave control, Rev. Mod. Phys. 89, 015005 (2017).

[54] S. Hemmady, X. Zheng, E. Ott, T. M. Antonsen, and S. M. Anlage, Universal Impedance Fluctuations in Wave Chaotic Systems, Phys. Rev. Lett. 94, 014102 (2005).

[55] S. Hemmady, X. Zheng, T. M. Antonsen Jr, E. Ott, and S. M. Anlage, Universal statistics of the scattering coefficient of chaotic microwave cavities, Phys. Rev. E 71, 056215 (2005).

[56] A. Z. Genack, P. Sebbah, M. Stoytchev, and B. A. Van Tiggelen, Statistics of Wave Dynamics in Random Media, Phys. Rev. Lett. 82, 715 (1999).

[57] M. Davy, Z. Shi, J. Wang, X. Cheng, and A. Z. Genack, Transmission Eigenchannels and the Densities of States of Random Media, Phys. Rev. Lett. 114, 033901 (2015).

[58] R. Savo, R. Pierrat, U. Najar, R. Carminati, S. Rotter, and S. Gigan, Observation of mean path length invariance in lightscattering media, Science 358, 765 (2017).

[59] M. Durand, S. M. Popoff, R. Carminati, and A. Goetschy, Optimizing Light Storage in Scattering Media with the Dwell-Time Operator, Phys. Rev. Lett. 123, 243901 (2019).

[60] P. del Hougne, R. Sobry, O. Legrand, F. Mortessagne, U. Kuhl, and M. Davy, Experimental realization of optimal energy storage in resonators embedded in scattering media, Laser Photonics Rev. 15, 2000335 (2021).

[61] P. del Hougne, K. B. Yeo, P. Besnier, and M. Davy, Ondemand coherent perfect absorption in complex scattering systems: Time delay divergence and enhanced sensitivity to perturbations, Laser Photonics Rev. TBA, 2000471 (2021).

[62] Y. Zheng, J. Huang, T. Chen, Y. Ou, and W. Zhou, Processing global and local features in convolutional neural network $(\mathrm{CNN})$ and primate visual systems, in Mobile Multimedia/Image Processing, Security, and Applications 2018 (International Society for Optics and Photonics,
Bellingham, 2018), Vol. 10668, p. 1066809, https://doi .org/10.1117/12.2305421.

[63] C. Zhu, E. A. Chan, Y. Wang, W. Peng, R. Guo, B. Zhang, C. Soci, and Y. Chong, Image reconstruction through a multimode fiber with a simple neural network architecture, Sci. Rep. 11, 896 (2021).

[64] T. J. Cui, M. Q. Qi, X. Wan, J. Zhao, and Q. Cheng, Coding metamaterials, digital metamaterials and programmable metamaterials, Light Sci. Appl. 3, e218 (2014).

[65] N. Kaina, M. Dupré, M. Fink, and G. Lerosey, Hybridized resonances to design tunable binary phase metasurface unit cells, Opt. Express 22, 18881 (2014).

[66] M. Dupré, P. del Hougne, M. Fink, F. Lemoult, and G. Lerosey, Wave-field Shaping in Cavities: Waves Trapped in a Box with Controllable Boundaries, Phys. Rev. Lett. 115, 017701 (2015).

[67] O. I. Lobkis and R. L. Weaver, On the Larsen effect to monitor small fast changes in materials, J. Acoust. Soc. Am. 125, 1894 (2009).

[68] P. del Hougne, M. F. Imani, A. V. Diebold, R. Horstmeyer, and D. R. Smith, Learned integrated sensing pipeline: Reconfigurable metasurface transceivers as trainable physical layer in an artificial neural network, Adv. Sci. 7, 1901913 (2020).

[69] H.-Y. Li, H.-T. Zhao, M.-L. Wei, H.-X. Ruan, Y. Shuang, T. J. Cui, P. del Hougne, and L. Li, Intelligent electromagnetic sensing with learnable data acquisition and processing, Patterns 1, 100006 (2020).

[70] B. W. Frazier, T. M. Antonsen, S. M. Anlage, and E. Ott, Deep wavefront shaping: Intelligent control of complex scattering responses with a programmable metasurface, arXiv:2103.13500.

[71] Y. Li, L. Li, L. Zhu, K. Maslov, J. Shi, P. Hu, E. Bo, J. Yao, J. Liang, L. Wang et al., Snapshot photoacoustic topography through an ergodic relay for high-throughput imaging of optical absorption, Nat. Photonics 14, 164 (2020).

[72] Y. Li, T. T. Wong, J. Shi, H.-C. Hsu, and L. V. Wang, Multifocal photoacoustic microscopy using a singleelement ultrasonic transducer through an ergodic relay, Light Sci. Appl. 9, 135 (2020).

[73] J. Bertolotti, E. G. Van Putten, C. Blum, A. Lagendijk, W. L. Vos, and A. P. Mosk, Non-invasive imaging through opaque scattering layers, Nature (London) 491, 232 (2012). 